\title{
How Data Access, Manipulation, Query, and Storage Has Transformed Overtime: A Study Based on User Perspective
}

\author{
Kelly R. Wibbenmeyer ${ }^{1}$ \\ ${ }^{1}$ Business School, Northcentral University, USA \\ Correspondence: Kelly R. Wibbenmeyer, Business School, Northcentral University, USA. E-mail: \\ kellyjason682002@yahoo.com
}

Received: June 1, 2015

Accepted: July 2, 2015

Online Published: August 22, 2015

doi:10.5539/ijbm.v10n9p79

URL: http://dx.doi.org/10.5539/ijbm.v10n9p79

\begin{abstract}
The purpose of this research is to provide practioners with information from the perspective of end users, database administrators, and developers regarding concerns and benefits of data management, data access, data manipulation, data query, and data storage in today's workplace. Synergy between data managers and end users is also a topic of discussion in this paper. The research results find that end users requirements are growing, organizational data needs are expanding, and database administrators and developers have more data challenges due to the increase of data security requirements and administrative processes within organizations. Due to the increasing data needs, end users are becoming more adept in creating their own reports. The reporting techniques available to end users must ensure data security and integrity standards are in place. Developers of graphical user interface (GUI) tools are responsible for safeguarding data. Database administrators and developers must always think of data security throughout the entire lifecycle of the data when coding applications to ensure data security is consistent, even after it leaves the premise. Cloud computing and big data is also an important aspect of data management in the future. An examination of cloud computing and big data constructs on existing data is also important as organizations adopt new technologies.
\end{abstract}

Keywords: data access, data query, data reporting, data manipulation, data storage, data synergy practices

\section{Introduction}

Technology continues to change at a rapid pace. Databases continue to optimize performance mechanisms that enable more data to be stored and processed (Rao et al., 2012). A study has estimated that the amount of information throughout the world increases exponentially at a rate of doubling every 20 months (Rao et al., 2012). Data increases lead to the need for additional data capacity, which leads to an overall increase in databases worldwide (Rao et al., 2012). This paper will address user's perspective regarding managing, accessing, querying, and manipulating data within a database. In order to obtain information for this paper, questionnaire data from six experienced database users is extrapolated and presented. This paper is important from a practioners perspective as real issues and perspectives from various database personnel, such as database administrators, query writers, and integration specialists, is presented.

\section{Literature Review}

Organizational data needs are growing at an exponential rate (Smith, 2012; Widmer, 2010). Technology changes daily to accommodate the needs of individuals and businesses to manage data access and storage needs (Ali, 2014). Cyber security is becoming a bigger issue as increases in the amount of data make it more difficult to manage the data needs ("Investment managers must", 2015). Organizations must have a data strategy in place prior to implementation of a data architecture to plan for data requirements ("Investment managers must", 2015). The use of data analytics is necessary to answer organizational questions that influence business decisions ("Investment managers must", 2015). As technology increases, the amount of data increases which increases the need for advancement in data storage, data availability, and data manipulation (Barlow, 2014).

Cloud computing technologies are becoming more prevalent as organizations need to find ways to manage data more effectively (Widmer, 2010). The transition from onsite to remote cloud computing and storage is occurring in organizations to increase organizational cost performance (Widmer, 2010). Cloud computing is a way organizations manage multiple access requirements while not drastically modifying the system architecture, 
which increases system efficiency (Widmer, 2010). Organizations that use cloud storage capabilities only purchase the storage they use which reduces overall storage costs (Widmer, 2010). Data redundancy occurs within the cloud, which reduces the time to replicate or retrieve data (Widmer, 2010). Multiple data risks occur when data is stored remotely (Widmer, 2010). If the cloud vendor goes out of business and the organization exclusively works with one cloud vendor, the organization can lose all access to data overnight (Widmer, 2010). Vendors do not want responsibility of maintaining security of the data within the cloud system (Widmer, 2010). Due to the increase in data, dashboards and other tools are needed to manage and monitor data risk and governance requirements within organizations ("PwC adds john", 2012). The transition to remote storage presents new organizational challenges, like risk mitigation for data security and data recovery procedures (Widmer, 2010).

Organizational efficiencies must be made to survive in the era of downsizing (Grohs, 2014). Technology advancements make it possible to do more with fewer people by transitioning paper processes to electronic processing (Grohs, 2014). Governmental regulations enforce the transition from paper to electronic means by requiring data access within a certain timeframe and to account for the number of data requests received (Hardwick, 2013).

Data management changes over time due to technology, cultural, and societal modifications (Mattia, 2011). Organizations must take measures to ensure compliance with data needs (Hardwick, 2014). Organizations have had to modify business practices, such as moving from paper to electronic sources, to keep up with customer demands (Hardwick, 2014). Data management and storage have undergone drastic modifications in the past decade (Opara \& Soluade, 2013). Organizations access data and manage data much differently now than they did in the past decade (Opara \& Soluade, 2013). Changes in data management, data storage, data access, and querying data practices can cause issues if the organization is not receptive to change (Opara \& Soluade, 2013). End users are playing a more active role in gathering their own data due to limited staff and data access needs ("International business machines, 2014).

\subsection{Diffusion of Innovation Theory}

The diffusion of innovations is a theory that examines the rationale why some organizations adopt technology quicker than other organizations (Hall, 2010). Organizations must be cognizant of technology modifications before the negative impact of not updating data structures becomes a detriment to the business practice (Hall, 2010). When organizations fail to plan for the adoption of technology, a loss of consumer confidence can occur and the business can lose market share (Shadam, 2015). The current paper will address the respondent's perceptions of adopting to technology modifications and how the modifications either negatively or positively influenced business operations.

\subsection{Study Background}

After a literature review of the issues surrounding increases in organizational data and how organizations are modifying practices to cope with the changes, responses from participants assist in obtaining a deeper understanding of the history of organizational data changes. The questions are open ended to gather as much information as possible from the respondents on the viewpoint of how organizations adapt to technology changes that impact organizational data. Participant questions deal with the perception of organizational change and how that change impacts business process, data management, and end user involvement. Businesses are dealing with a reduction of employees which makes it difficult to continue to run business as usual and modify technology concurrently (Opara \& Soluade, 2013). If organizations do not modify business practices to ensure data access, storage, and management needs are met a loss of market share and consumer confidence can ensue (Opara \& Soluade, 2013). Organizations are currently in a bind as consumers demand new technology and the business resources are now not available to keep up with the technology demands (Opara \& Soluade, 2013). The diffusion of innovations is a theory that examines what occurs when organizations do not modify technology to ensure the survival of the organizations and is relevant to the current study.

\subsection{User Background}

Six experienced database users were questioned regarding their experience using the database at their place of employment. An invitation to respond to 12 general IT questions went out to database users, super users, database administrators, query creators on a popular survey tool (survey monkey) was used to acquire answers within a two week period. The users were all asked the same twelve essay type questions dealing with managing, accessing, querying, and manipulating data (see Appendix 1 for the questions presented). The questions were open-ended to allow users to contribute based on their specific experience. Respondents were from various industries such as the travel, healthcare, utility, insurance, and communications industry. Users also varied based 
on age group, employment status (part-time, full-time) and consultant status. The one commonality between the users was they all use database management on a daily basis for work. The interviewees responded independent of each other in order to ensure each respondent's opinions are not influenced by the other interviewees. No clarifying follow-up questions were asked based on the initial survey submitted to the respondents.

\subsection{Transformation from Paper to Electronic Data}

Management of data has been transformed over the past few decades from a paper management practice to an electronic standard. The move from paper to electronic means has allowed databases to become more popular. One respondent remembers a time prior to computers when data was handwritten, and the usage of carbon (to duplicate documentation) was popular (Siedel, 2015). Widespread usage of photocopying was used to reduce re-keying, and early electronic records were maintained on punch cards or large magnetic floppy disks (Siedel, 2015). Computers were immediately useful as tasks such as complex calculations could be programmed in excel, which greatly reduced calculation time and allowed for real-time data (Van Rossler, 2015). Another respondent stated that computers reduced the amount of copy errors and enabled more expedient review of data (Kaiser, 2015). Tremendous increases in physical space saving occurred with the move from paper to electronic (Siedel, 2015). A respondent remembers filling large filing cabinets before floppy disks were available (Siedel, 2015). Another respondent doesn't remember a time prior to computers but states that data entry was first completed on mainframe systems (Wilson, 2015). Data entry was not easy as point and click technology was not yet introduced, and function keys had to be memorized for data entry, which lead to very cumbersome data entry and many data errors (Wilson, 2015). Data access, indexing, sorting, grouping, tabulation and cross-referencing of records is much easier for end users that have access to create reports (Siedel, 2015). Data processing increases drastically, and the amount of paper used in the organization decreases drastically with the switch to electronic processing (Wilson, 2015). Data movement is much more expeditious with electronic processing as there is no need to print paper, route the paper, and re-key data (Wilson, 2015). Data quality also increases with electronic means due to integration between systems instead of re-entering data (Wilson, 2015). Secure optical storage alleviates the need to print and maintain paper documents (Wilson, 2015). The movement from paper-based systems to electronic-based systems has impacted overall organizational processes over the years.

\section{Data Management and Storage}

Data management has changed drastically over the past decade ("Quest software addresses, 2011). Technology advancements have stimulated multiple data inputs in various formats (phone, computer, digital pens, scanning, and bar coding) ("Abstracts from the society, 2012). The amount of data available has also drastically increased over the last decade due to the various input mechanisms to capture and store data (Barlow, 2014; Van Rossler, 2015; Wilson, 2015). One of the interviewees stated that the amount of data has exponentially increased, such as capturing the data collection method (Wilson, 2015). Additional storage requirements are needed due to the granularity of the data requirements and the amount of data required (Kaiser, 2015; Van Rossler, 2015). In the past, organizations would only collect required form data, and all other data was disregarded; today all data is captured (Wilson, 2015). More data is being retained for data trending and demographic needs (Wilson, 2015). Linking data across multiple organizations is also becoming a standard practice that requires the data storage mechanism to maintain data from multiple inputs (Van Rossler, 2015). Increases in the amount of data needed by organizations to run effectively have drastically increased and, therefore, additional data storage is required to effectively manage the surge of information.

Organizations are looking for creative ways to add more storage capabilities to retain the additional data being kept (Wilson, 2015). Many organizations are moving to the cloud for their storage requirements (Wilson, 2015). Cloud based storage removes the requirement for organizations to store, secure, and backup data (Wilson, 2015). The issues associated with data storage and maintenance, such as ensuring policies are kept updated to remove potential security risks are reduced when data maintenance is completed on the cloud (Wilson, 2015). Many businesses are using cloud base technologies to reduce the risks associated with IT security updates (Opara \& Soluade, 2013). Enterprises use cloud base platforms to increase productivity by working on value added business specific activities instead of spending efforts on global system updates that need to occur (Opara \& Soluade, 2013). Cloud base businesses perform security updates on common platforms such as Internet Explorer patch updates, Windows security updates, and other normal system maintenance activities (Opara \& Soluade, 2013). Cloud based businesses perform these updates on a global level, where they perform the modification, test the modification and deploy the modification to all systems located within their cloud space (Opara \& Soluade, 2013). The wide scale updates completed at the cloud based company reduce disaster recovery time and increase business continuity activities (Opara \& Soluade, 2013). By employing cloud based services, organizations maximize their skilled talent by placing them on organization specific activities to enhance business processes 
(Opara \& Soluade, 2013). The cloud based approach increases overall productivity. Organizations not using the cloud, spend more money internally as they have resources dedicated to update routine maintenance that could be implemented by other resources.

Data standards have drastically increased, such as those imposed by regulatory requirements, especially over the past ten years (Shadam, 2015). The healthcare industry had to adapt to the Health Insurance Portability and Accountability Act (HIPAA) standards (Shadam, 2015). Sarbanes-Oxley (SOX) standards are implemented to reduce fraudulent activities (Shadam, 2015). Due to the ever-increasing data requirements, sensitive data must be kept secure. Securing data is a vital part of data operations in organizations. One of the major data requirement modifications over the past few years has been encrypting personally identifiable information (PII) due to potential security breaches (Siedel, 2015). Methods such as data encryption algorithms and redaction techniques are used to protect PII and other sensitive data such as pricing, client lists, copyrighted and patented info (Siedel, 2015; Van Rossler, 2015). Data regulations influence data management practices, such as data structure and what data is send to who and when it's sent. An example of the impact of data regulations on business practices is sending monthly documentation to governing bodies based on audit requirements (Shadam, 2015). Data is also being retained for longer timeframes due to legal aspects such as record retention requirements (Wilson, 2015). In order to deal with the increased demand for data, organizations are hiring more people, implementing more robust training programs, and increasing auditing and legal support to deal with potential breaches (Van Rossler, 2015). Regulation impacts the way data is managed and stored to extrapolate data when it is required and enforces who is allowed to view the data.

Business data needs are growing at a rapid rate. Organizations are requesting more data, both from an external and internal perspective (Kaiser, 2015; Shadam, 2015). As data requests are made, reports are generated to present the users with only the data requested (Shadam, 2015). In the past, users were given generic reports that were lined through (if they didn't have the rights to access the data) in order to present data in a timely manner (Shadam, 2015). Managing data has become easier, even though there is more data to manage due to more people becoming accustomed to electronic data entry (Kaiser, 2015; Shadam, 2015). On the other hand, the other interviewee thought managing data has become more difficult due to the amount of data required by organizations (McDunna, 2015). Even though user's had very similar database knowledge, the user who was responsible for writing more difficult queries without a GUI interface (McDunna) found the data management portion to be more difficult due to the amount of data that is being parsed on a daily basis. Data retrieval was not a concern for another respondent (Shadam) as he used a GUI interface with a defined set of tables to extract data. The majority of data management concerns were based on the amount of data retrieved, the method of retrieval (GUI interface or manually writing reports), and the frequency of retrieval.

\subsection{Data Access}

Transformative data access strategies implemented throughout the past decade modify data requirements and availability ("Solar company skyline", 2010). Data access varies based on the user perspective; this section delineates between the end user perspective and the data administrator's perspective, as they are vastly different. Data access has been drastically improved for users over the past decade (Shadam, 2015). Data access has become much easier from a user's perspective due to more robust user friendly tools for searches and reporting (Van Rossler, 2015). The timeframe for obtaining access to the data is much quicker as well. There was a time where it took a few weeks to a month to obtain database access; then training took another month to complete (Shadam, 2015). Data access for end users has drastically enhanced over the past decade.

Data access, from a data administrator's point of view, has become much more complicated due to additional security requirements (Seidel, 2015; Van Rossler, 2015; Wilson, 2015). The amount of "red-tape" database administrators and developers have to go through impacts the timeliness of the query (Van Rossler, 2015). Data access must be secured. Within the past few years, additional requirements such as securing the code that accesses the data must also be taken into account (Wilson, 2015). Fewer users have access to the data today than years ago in order to keep users' data secure (Van Rossler, 2015; Wilson, 2015). The more information that is stored within the organization, the more controls must be in place to lock down the data (Wilson, 2015). Today, restricted access is required which includes restricting data views of specific record types and fields or restricting the ability to download and print confidential data such as credit cards or passwords (Seidel, 2015). Securing data from breaches has been a top priority to most organizations due to the high profile cases that have been in the news (such as credit fraud and identity theft) (Seidel, 2015). Securing access points, encryption of confidential data and restricting access to only those who need access at the time data is needed and only what is needed is more closely scrutinized than ever before due to liability risks (Seidel, 2015). The additional security measures take additional time due to additional coding and coordination that must occur within the team (Wilson, 
2015). During the creation of applications, the developer must take security risks into account (Wilson, 2015). Technology employed by developers has increased which eases some of the burden associated with dealing with the mitigation of security risks (Wilson, 2015). Certain database fields are locked down based on user permission rights; this technology not available a decade ago (Shadam, 2015). Permission-based data tables allow for additional data retrieval that was previously unavailable due to confidential data fields and, therefore, the entire table was not available (Shadam, 2015). With the new technology, users have the ability to view data based on current need (Shadam, 2015). The access rights to specific fields or entire tables can also be driven by accessibility timeframes that restrict access after a certain time period is over (Shadum, 2015). Since the database handles security on a field by field basis, a more robust data strategy is available (Shadam, 2015). Data access has greatly improved for end users and has grown more complicated for developers over the past decade.

\subsection{Querying the Database}

Query optimizers assist in speeding up results retrieval and maximizes the database performance ("International business machines, 2014). Data restrictions continue to increase, and therefore reports require additional security based on who should have access (Siedel, 2015; Wilson, 2015). Reporting used to be a one-report-fits most approach and recently has been modified to who should see the report and who can see what data fields on the report (Wilson, 2015). In some cases even users who have rights to view the data cannot see the data without redaction techniques in place as someone else may view the data while it's in printed form (Siedel, 2015; Wilson, 2015).

A decade ago, user requirements were much simpler (Van Rossler, 2015). In today's data driven society, users require reporting methods that allow them to specialize the output based on need (Kaiser, 2015; Van Rossler, 2015). Executives require dashboards with easy-to-read graphics that can drill down into specific metrics which entails more complex reporting methods (Kaiser, 2015; Van Rossler, 2015). Organizational processes has dictated a more intricate level of data security, which has led to creating more reports, each with their own specific set of data and security requirements (Wilson, 2015).

Basic reporting is much easier than it was over a decade ago (Shadam, 2015; Wilson, 2015). Reporting mechanisms are simplified today due to friendlier user interfaces. Reporting tools have significantly improved, which allows for easier creation and manipulation of reports for both the developer and end user (Shadam, 2015; Wilson, 2015). The increased demand for reporting has evolved into more experienced users creating their own reports (Kaiser, 2015; Wilson, 2015). One downside of users creating their own reports is the potential for unreliable reporting (Kaiser, 2015). With the abundance of GUI tools, inexperienced users are building reports and the data inputs are not confirmed. Therefore, critical reports should be created by IT (Kaiser, 2015). As users start generating more reports, fewer report requests are submitted to the developer, which expedites report creation and reduces costs in building reports (Wilson, 2015).

Advances in hardware and querying algorithms allows for quicker data retrieval methods (Wilson, 2015). Computer processing speeds enable faster queries to occur against larger databases with more data (Van Rossler, 2015). A report that use to take hours to run and maximize system resources a decade ago can complete within a matter of seconds today (Van Rossler, 2015). Real-time data enables organizations to make better decisions due to more timely data (Wilson, 2015). Companies are requiring real-time, complex data processing mechanisms to be in place in order to run their business more efficiently (Van Rossler, 2015).

Organizations are required to secure confidential information such as pricing, suppliers, clients, patents, copyrighted material (Seidel, 2015). When the confidential information is located in queries online, it's important that only users with the appropriate access can view the data. Therefore, the data that gives organizations the competitive advantage is sometimes the hardest to maintain and secure (Seidel, 2015). Obtaining the data to create reports, for both developers and end users, is easier but the additional required security measures makes the reporting more complicated from a developer standpoint.

\subsection{Manipulate Data}

Data manipulation is easier to perform with the vast amount of tools available for the end user. Data manipulation from a developer or database administrator is much more difficult, due to the additional administrative processes that are required to ensure security measures in place (Wilson, 2015). The actual data modification process is fairly straightforward for the developer (Wilson, 2015). Additional tools are available to manipulate data easier than ever before (Wilson, 2015). SQL is an easy way to modify data in bulk for the developer ("International business machines, 2014). Scripting languages allow the user to modify data based on attributes associated with data via mass system operations. Data is more accessible and easier to update when bona-fide users access the system (Kaiser, 2015; Seidel, 2015). Data manipulation can be difficult when 
inexperienced users are attempting to find the data in the system due to the amount of data to parse through (Van Rossler, 2015). Robust query tools allow organizatons to find the data to manipulate (Van Rossler, 2015). Since reporting is so much easier in today's world, data is more readily available for consumption (Kaiser, 2015). Data corruption or falsifying data is much more difficult for unauthorized users due to increased regulations regarding data security (Seidel, 2015).

The biggest roadblocks for manipulating data from a developer perspective is working through the administrative process, such as working with other departments, such as the database administrators to obtain approval to make the modification or the multiple levels of auditing in place (Wilson, 2015). These additional requirements add complexity, especially during the identification phase where the developer is attempting to figure out the solution (Wilson, 2015). Additional data requirements have pushed organizations to modify their data strategies and hire data analyst teams to manage information (Kaiser, 2015). In the past, the developer could make a modification to the code and submit it to the system, test it out, and if it didn't work update the code and try again (Wilson, 2015). In today's complex world, every program modification must go through appropriate auditing channels, which adds tremendous amounts of time when fixing issues (Wilson, 2015).

Auditing efforts have increased due to regulations; this has caused an increase in awareness of the user's perspectives (Shadam, 2015). Users are reviewing data for accuracy and, therefore, the need to modify data has increased (Shadam, 2015). Based on user requirements, various user interfaces are available that can integrate with data from various sources. Some applications have a web-based, and application-based application that integrate to the same database and the user can select which option best fits their requirements (Shadam, 2015). Developers also create user-facing web applications to meet a specific need, such as filling out form data, which make data manipulation much easier for the end user. The specific web applications increase the ability to update and manipulate a limited data scope, which in turn makes it easier to enter the data (Shadam, 2015). Creative data manipulation strategies can be presented such as mass updates to data or the creation of a GUI web-based application to allow for easy data manipulation for the end user. Auditing education throughout the organization has increased which results in a workforce that is knowledgeable in data regulation (Shadam, 2015).

\subsection{Big Data and Cloud Computing}

The most recent data analysis methodology is big data analysis (Purcell, 2014). Big data is possible due to technology advancements that allow huge data storage and analysis capabilities (Shadam, 2015). Traditional database systems are not able to store and analyze the large datasets that are available with the introduction of big data (Purcell, 2014). Data requirements are increasing as multiple data sources such as e-mail, documentation, correspondence, and social media inputs are now required to be kept within the organizations data repositories for easy retrieval (Shadam, 2015). Multiple organizational challenges are present due to the storage and analysis concerns present with big data (Purcell, 2014). Big data can be very cost prohibitive especially to small businesses as the number of processing resources to maintain big data is vast (Purcell, 2014). The introduction of cloud computing allows small businesses the opportunity to use big data as outside resources perform the data computation (Purcell, 2014). All businesses can increase their productivity by moving to cloud computing services (Purcell, 2014). Businesses decrease the hardware and processing costs when moving to cloud computing services (Purcell, 2014). Security threats and loss of data control are the primary concerns for organizations when thinking about moving to cloud computing services (Purcell, 2014). Therefore, organizations that have restricted data or sensitive data must ensure appropriate measures are in place prior to moving to a cloud based data strategy (Shadam, 2015).

\subsection{User and Developer Synergy}

User and Developer synergy is vital to organizations. Failure to understand end user processes and how those processes relate to the system update will result in rework for the developer and frustration for the end user (Van Rossler, 2015). Developers will also lose their credibility with the end user due to the lack of understanding the process (Van Rossler, 2015). The lack of understanding between end users and developers is typically due to a lack of effective communication (Kaiser, 2015; Van Rossler 2015; Wilson, 2015). Developers are responsible for ensuring the integrity and security of confidential data and design applications with these requirements in mind while providing data access that meets the end user requirements (Seidel, 2015). Developers require an in-depth knowledge of the database in order to create complex queries. Interfacing with other systems is another task developer's perform on a regular basis. Developers must be able to manipulate and mitigate data concerns. Due to the requirement to modify data, developers must have an extensive knowledge of the data, how it's interconnected, and the effects analysis of moving and modifying data. The user provides the details surrounding what data is needed when it is needed, and who needs access to the data (Seidel, 2015). The minimum access 
requirements are obtained from the end users, and the developers create the mechanism and ensure the requirements are met (Seidel, 2015).

Developers have administrative rights over the data and, therefore, are responsible when data modifications cause system issues. Data modifications are closely monitored in large databases that have multiple users since some data modifications can corrupt the database. Consumers of databases require enough database knowledge in order to ensure basic reporting is completed using the appropriate fields (to ensure appropriate and accurate reporting) (Van Rossler, 2015). The more database knowledge users acquire, the better the user understands the system complexity. As users gain more database knowledge and experience, they perform additional general system duties, and, therefore, the developer's workload is reduced. Some of the general system duties users can perform is writing basic reports (usually via a report writing tool) and modifying data. Synergy between users and developers ensure a greater chance of task attainment (Kaiser, 2015; Tesch et al., 2009). Faulty requirements gathering is a result of teams that do not work well together. When the developer does not obtain appropriate requirements, more time is spent working on quality issues due to the lack of appropriate requirements (Van Rossler, 2015). A developer can build the best application which turns out to be terrible for the users if the users don't use the system in the way the developer intended (Van Rossler, 2015; Wilson, 2015). "Something as simple as the order in which the fields are placed on the page can have a tremendous effect on how the application works from an end users viewpoint" (Wilson, 2015). If the developer and end user exhibit effective communication, the result is a much better end product (Kaiser, 2015; Wilson, 2015).

Based on the information collected in the questionnaires, database end users understand the basic reporting tools and database concepts, such as tables and fields. The users were able to create queries and update data as long as they had the appropriate permissions. The users similarly understand what data they are allowed to modify and what data is secured and why these system controls are in place. Additional communication and collaboration between developers and users would give the developers additional insight into business processes (Tesch et al., 2009). Understanding the business processes would enable the developer to gain additional background information for the purpose of advanced data queries and assist in solving problems (Tesch et al., 2009).

\section{Discussion}

The respondents consistently agree that technology changes impact business processes over time. Users are more technically savvy today, then they were just a decade ago. This increases the likelihood that end users perform more data analysis themselves. A decrease in employees available to manage data also impacts current business processes as more users must perform their own data management if data needs are immediate (Opara \& Soluade, 2013). Developers must continue to find creative ways to ensure data reliability while handing the majority of data querying efforts over to end users. As users continue to become more technically savvy, understanding more complex data constructs will become even easier to understand, which will aid in better synergy between the end user and the developer. Allowing users with more technical experience the ability to manipulate data may also alleviate some of the workload of the developer, but this modification will not come without risk. Some issues present with giving end users additional access are data risks, such as data corruption or data theft. Another major concern with granting end users access is the likelihood of invalid data increases as the end user may retrieve the incorrect data within their query. Data access and storage strategies continue to evolve over time and will most likely continue to change as organizational needs dictate transformation.

\section{Conclusion}

In summary, data has changed drastically over the past few years. The transformation from paper to electronic methods has transformed organizational processes, which has enhanced productivity. Drastic increases in data results in additional storage needs and sparks creative solutions to manage the excessive amounts of data. The use of cloud based technology to manage system maintenance is one method of organizational efficiency to ensure data is secure. Increasing data standards also push organizations to become more efficient as more organizations are doing more (due to more data to manage and more regulatory requirements) with fewer resources. Data access has become easier for users due to advances in technology, but more challenging for developers due to increasing standards for data security. Organizations require more immediate data. Due to increasing data security policies, most businesses prepare custom reports that are end user specific. The need for additional data has encouraged end users to create their own reports on an as needed basis. As end users create more reports on their own, developers are spending more time working on critical or more challenging reports, which also increases productivity within an organization. Data manipulation has become easier for both the end user and developer due to easy to use tools. There are new pain points for developers regarding data manipulation due to the additional restrictions for modifying data, which ensure the appropriate developers are 
modifying the data at the appropriate time. Developer and user collaboration will streamline operational efficiencies and reduce quality errors. The diffusion of innovation theory constructs are present in the respondent's answers as the benefits and risks of transitioning to the new technology, when the transition was made, and how the technology advancements impacted business practices greatly influenced data practices. Interviewing database consumers is useful in understanding the changes in data management, reporting, access and manipulation and how those modifications have affected business operations within organizations.

\section{References}

Ali, M. (2014). How 3g will change our lives. Flare, 10, 20. Retrieved from http://cms.uflib.ufl.edu/flare/Index.aspx

Barlow, R. D. (2014). Big data becoming a mined-over matter. Health Management Technology, 35(5), 6-9. Retrieved from http://www.healthmgttech.com/2015/

Grohs, M. (2014). Court management conference. Courts Today, 12(4), 4-12. Retrieved from https://www.nacmnet.org/

Hall, G. E. (2010). Technology's achilles heel: Achieving high-quality implementation. Journal of Research on Technology in Education, 42(3), 231-253. http://dx.doi.org/10.1080/15391523.2010.10782550

Hardwick, D. (2013). Regulatory changes demand an enterprise-wide approach to disclosure management of PHI. Journal of Health Care Compliance, 15(4), 17-22, 65-66. Retrieved from http://www.hcca-info.org/

Kaiser, J. (2015). Interview by K. Wibbenmeyer [Questionnaire].

Mattia, A. (2011). An exploratory study on using stage theory to develop a data management measurement framework. International Journal of Management and Information Systems, 15(4), 123-132. Retrieved from http://www.cluteinstitute.com/journals/international-journal-of-management-information-systems-ijmis/

McDunna, K. (2015). Interview by K. Wibbenmeyer [Questionnaire].

Opara, E. U., \& Soluade, O. A. (2013). Effect of virtualization on enterprise network, Server/Desktop systems on small and mid-size businesses (SMB). Journal of International Technology and Information Management, 22(2), 35. Retrieved from http://www.iima.org

Purcell, B. M. (2014). Big data using cloud computing. Journal of Technology Research, 5, 1-8. Retrieved from http://www.aabri.com/jtr.html

PwC adds john sabatini to risk assurance practice. (2012). Wireless News. Retrieved from http://www.pwc.com/us/en/index.jhtml

Rao, C. S., Kumar, G. Y. R., Reddy, G. P., \& Kumar, R. B. (2012). Optimizing data mining tasks using rbf neural networks. International Journal of Marketing and Technology, 2(11), 30-39. Retrieved from http://www.inderscience.com/jhome.php?jcode=ijtmkt

Shadam, J. (2015). Interview by K. Wibbenmeyer [Questionnaire].

Siedel, D. (2015). Interview by K. Wibbenmeyer [Questionnaire].

Smith, R. (2012). Bay area: In san leandro, a drive to get wired. Wall Street Journal. Retrieved from http://www.wsj.com/

Tesch, D., Sobol, M., Klein, G., \& Jiang, J. (2009). User and developer common knowledge: Effect on the success of information system development projects. International Journal of Project Management, 657-664. http://dx.doi.org/10.1016/j.ijproman.2009.01.002

Van Rossler, M. (2015). Interview by K. Wibbenmeyer [Questionnaire].

Widmer, L. (2010). The cloud and your data. Risk Management, 57(9), 36-40. Retrieved from http://www.rmmagazine.com/

Wilson, T. (2015). Interview by K. Wibbenmeyer [Questionnaire].

\section{Appendix 1- Questions sent to Respondents}

1) What industry do you work in?

2) What is your role (developer, database administrator, end user, query writer, etc)? 
3) What was data entry like prior to computers (if you can't remember a time before computers please state this and state what it was like when you were first introduced to computers)?

4) What are the greatest impacts to electronic society with the transition from paper processing to electronic processing?

5) How have data requirements changed in the past ten years?

6) How have organizations morphed to deal with the ever changing data requirements?

7) How have reports modified in the past ten years? (think about this question from the perspective of data access)

8) How has data access changed in the past ten years? (more complicated /less complicated)

9) How has data management (data retrieval (querying technology (aka reporting)), data input, data storage, data access) practices changed from an organizational perspective in the past decade?

10) Is it easier or more difficult to manipulate data with today's world? Why?

11) How has data access policies changed over the past ten years?

12) Many users believe that developer and user synergy is important. Why?

\section{Copyrights}

Copyright for this article is retained by the author(s), with first publication rights granted to the journal.

This is an open-access article distributed under the terms and conditions of the Creative Commons Attribution license (http://creativecommons.org/licenses/by/3.0/). 